\title{
The Parameters Optimization of the Clamp Locking Mechanism
}

\author{
Ai Xiayu ${ }^{1}$, Shi Xiang ${ }^{1, a}$ and Zhang Zhen ${ }^{1}$ \\ ${ }^{1}$ College of Engineering, Ocean University of China, Qingdao 266100, China \\ ashixiang331@vip.sina.com
}

\begin{abstract}
Keywords: clamp; locking mechanism; parameters optimization; finite element analysis
Abstract. Leak repair clamp is the preferred method for permanently repairing pinhole and crack-type pipeline leaks underwater, and the locking mechanism is a key component of it. In this paper, through the analysis on the bite-in theory and the failure mode of the locking mechanism of clamp, three principles for design of the locking mechanism, which are the principle of the contact stress uniformity of the teeth, the principle of axial bearing capacity and the principle of sufficient jacking allowance, are proposed. Then using related principles and finite element analysis, the structural parameters of the locking mechanism, including the slip anchor teeth thread angle, the wedge angle of the inner and outer slip anchors as well as the front and rear angles combination of the thread angle, are optimized. The recommended thread angle is $80^{\circ}$, the recommended wedge angle of the inner and outer slip anchors is $13^{\circ}$, and the recommended front and rear angles combination of the thread angle is $65^{\circ}$ and $15^{\circ}$.
\end{abstract}

\section{Introduction}

China has successively built 47 oil and gas fields in different sea areas. There are hundreds of pipelines of various specifications with a total length of more than $6,000 \mathrm{~km}^{[1]}$. Some of them have been used for more than 15 years, and some have even worked for 25 years ${ }^{[2]}$. A considerable proportion of submarine pipeline has to face serious aging and will exceed the design life. Coupled with the frequent occurrence of global disaster climate, the probability of damage and leakage of submarine pipelines has increased dramatically. Therefore, it is necessary to develop pipeline maintenance technology. The clamp is a key component of the submarine pipeline maintenance technology. It can be used to repair the pipeline with minor damage, such as local mechanical damage, corrosion piercing, cracks, axial or circumferential dents, etc. And it can achieve permanent sealing and is easy to install ${ }^{[3]}$. Over 30 years of research has been conducted on the repair technology of clamp in foreign countries. More than 3,000 clamps have been installed in the shallow sea area. The reinforced pipe diameter ranges from 3 inches to 48 inches and the plugging pressure can reach 25 $\mathrm{MPa}^{[4]}$. The domestic clamp technology is relatively backward, and there is at least 15-20 years of technology gap with foreign technology. The main domestic products are temporary maintenance clamps, which cannot be used without stopping the delivery, and their underwater installation is inefficient. When there is a pipe leak and a foreign clamp has to be used for repair, the maintenance cost is huge ${ }^{[5]}$. The function of the locking mechanism of the clamp is to connect the clamp structure with the damaged pipe to provide structural strength, and the locking effect directly determines the success or fail of the maintenance ${ }^{[6]}$. The locking mechanism is driven by the wedge principle, and the purpose of locking is achieved by the teeth biting into the pipe.

Through the analysis on the bite-in theory and the failure mode of the locking mechanism of clamp, this paper aims to put forward the design principle of the locking mechanism. And using related principles and finite element analysis, the parameters of the locking mechanism, including the slip anchor teeth thread angle, the wedge angle of the inner and outer slip anchors as well as the front and rear angles combination of the thread angle, are optimized.

\section{Theory of the locking mechanism}

Through years of research abroad, a consensus has been formed on the overall structure of clamps ${ }^{[4]}$ [7]. The pipe clamp structure mainly includes two upper and lower clamp housings, main bolts connecting the two housings, a pair of jacking flanges and clamp locking mechanisms on both sides. 
The locking mechanism of the clamp is composed of inner slip anchor, outer slip anchor and baffle. The specific structure of the clamp is shown in Figure 1.

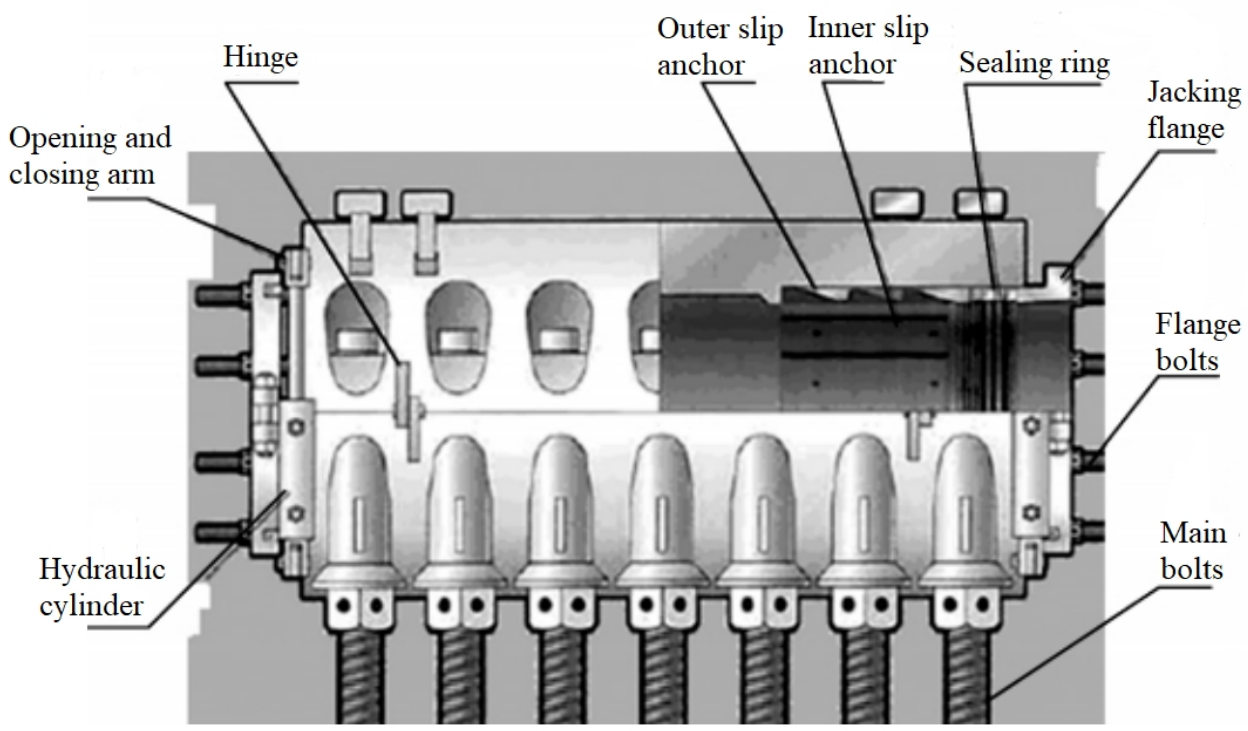

Figure 1 The specific structure of the clamp

The working principle of the locking mechanism (shown in Figure 2) is that the jacking flange exerts an axial force on the outer slip anchor, which drives the outer slip anchor to move axially. The outer slip anchor drives the inner slip anchor through the wedge principle to make radial movements. At last, the teeth on the inner slip anchor bite into the pipe wall, and the teeth on the outer slip anchor bite into the clamp housing wall to achieve the effect that the clamp locks the pipe.

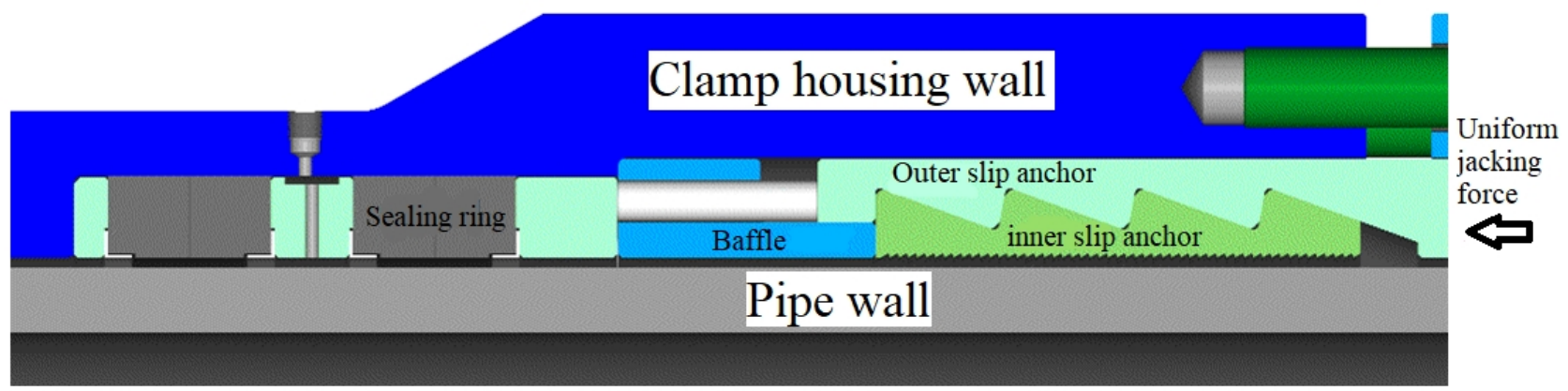

Figure 2 The working principle of the locking mechanism

\section{Mechanics of the locking mechanism}

Calculation method of bite width. By analysis on the wedge principle for locking mechanism, the squeezing force $N$ of each inner slip anchors to the pipe can be obtained. Assuming that the squeezing force is evenly distributed on each tooth, the biting force $F$ of the single tooth on the pipe is

$$
F=\frac{N}{n_{1}}
$$

Where $n_{1}$ is the number of teeth on a single slip anchor.

According to Han Guangyao's research ${ }^{[8]}$, the bite force of a single tooth meets the following formula: 


$$
F=\sigma_{s} b \frac{1}{\tan \alpha+\tan \beta}\left(\frac{1}{\cos \alpha}+\frac{1}{\cos \beta}\right) L
$$

Where $\sigma_{s}$ is the yield strength of pipe wall, $b$ is the bite width, $\alpha$ is the front angle, $\beta$ is the rear angle, and $L$ is the circumferential length of the teeth, as shown in Figure 3.

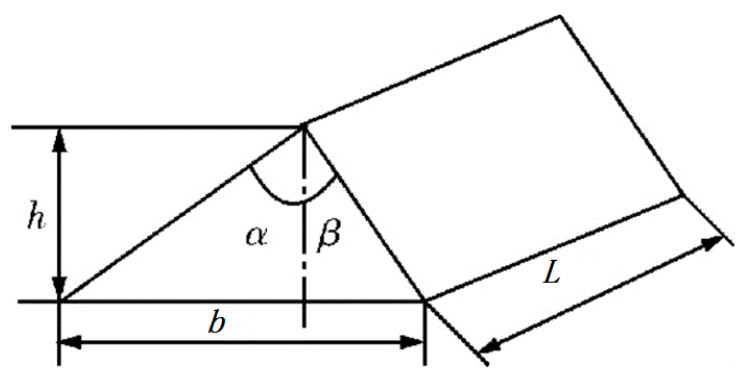

Figure 3 The geometry parameters of a tooth

This gives the expression of bite width

$$
b=\frac{N}{n_{1}} /\left[\sigma_{s} \frac{1}{\tan \alpha+\tan \beta}\left(\frac{1}{\cos \alpha}+\frac{1}{\cos \beta}\right) L\right]
$$

Failure mode. A failure mode of the clamp is that the parts of teeth biting into the pipeline are all sheared to be broken under the axial external force. And the locking mechanism is failed to work. According to the failure mode, the axial bearing capacity of the clamp is

$$
N^{\prime}=n_{2} \pi D_{p_{o}} b[\tau]
$$

Where $N^{\prime}$ is the axial bearing capacity of the clamp, $n_{2}$ is the number of teeth on inner slip anchor around the pipe, $D_{P Q}$ is the outer diameter of the pipe, $b$ is the bite width, and $[\tau]$ is the shear strength of the teeth.

\section{Design Principles of the Locking Mechanism}

Principle of the contact stress uniformity of the teeth. As the key structure of the locking mechanism, the inner and outer slip anchors are wedge-shaped structures. And different biting depths of teeth for slip anchors will not be conducive to exerting the axial bearing stability of the locking mechanism. Therefore, a design principle is stipulated: the contact stress of each tooth should be as uniform as possible at the beginning of the bite so as to ensure that the bite depth of each tooth is not much different.

Principle of axial bearing capacity. The clamp is generally suitable for the repair of pipelines with minor damages, such as large local mechanical damage, perforation, cracks, axial or circumferential dents, etc. In these cases, the pipeline itself still retains a large residual strength. Therefore, the designed axial bearing capacity of the clamp does not need to equal the breaking load of the intact pipe. For the sake of simplification, it is stipulated in the design principle: The designed axial bearing capacity $N^{\prime}$ of the locking mechanism should be greater than half of the intact pipe tension breaking load $T$. It is expressed as:

$$
N^{\prime} \geq 0.5 T
$$

Principle of sufficient jacking allowance. The locking mechanism starts to bite in the pipe when the circumferential centering of the clamp is completed. According to the working principle of the locking mechanism, during the process when the teeth bite into the pipe wall and the clamp housing 
wall, the outer slip anchor will axially move under the axial jacking force. In order to ensure the bite depths, the following design principles are stipulated: a sufficient jacking allowance is reserved between the baffle and the outer slip anchor after completion of clamp centering.

\section{Basic model dimensions and their determinations}

The pipe with outer diameter of $508 \mathrm{~mm}$ and thickness of $20 \mathrm{~mm}$ was selected to design the clamp, and the thickness of the clamp housing was set to be $30 \mathrm{~mm}$. The distance between the inner wall of the clamp housing and the outer wall of the pipe was $31 \mathrm{~mm}$. The inner slip anchor has the same length as the outer slip anchor. The lengths of the slip anchors were set to be $114 \mathrm{~mm}$ first. The teeth were made on the slip anchors. Figure 4 shows the state where the slip anchors have just completed the clamp centering.
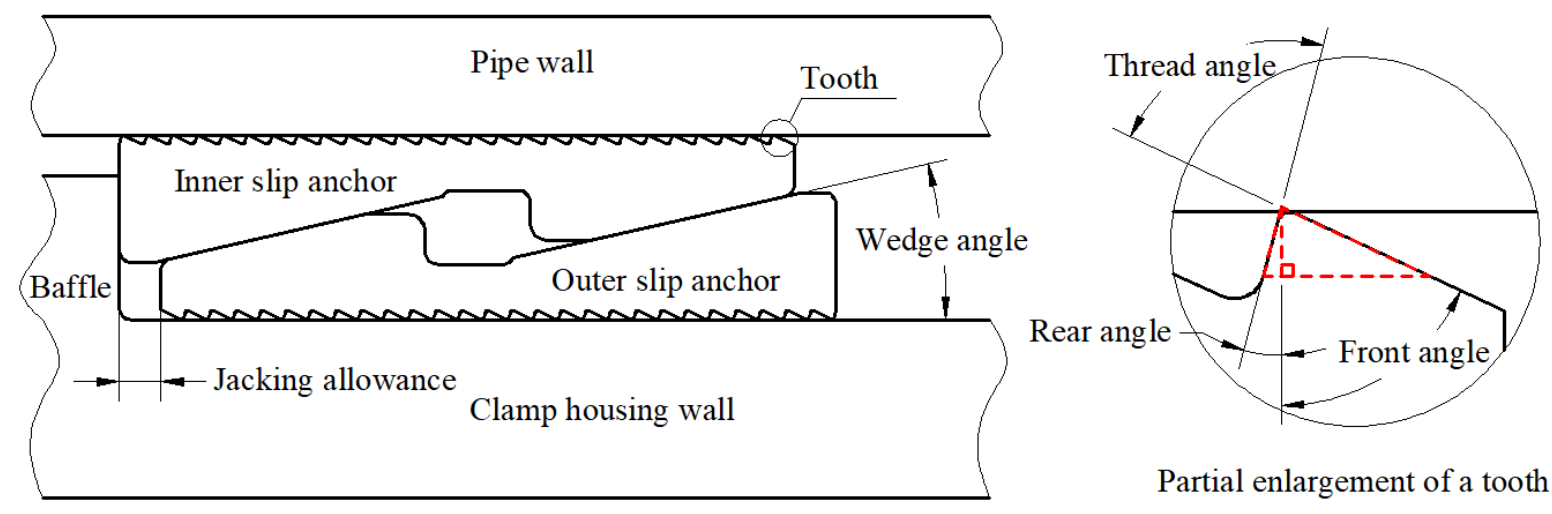

Figure 4 Schematic diagram of the components of the locking mechanism

Determination of the thread angle. A failure mode of the clamp is that the portion of the teeth biting into the pipe is sheared to be broken under the axial external force, so the bite width is positively related to the bearing capacity of the clamp. In order to ensure a sufficient bite width, the thread angle of the teeth should not be too small. Combined with the relevant component design experience, the three angles of $80^{\circ}, 90^{\circ}$ and $100^{\circ}$ were preliminarily selected, and the number of isosceles teeth that can be machined on slip anchor was calculated (see Table 1).

Table 1 The number of teeth that can be machined

\begin{tabular}{cccc}
\hline Thread angle $\left[{ }^{\circ}\right]$ & Tooth height $[\mathrm{mm}]$ & Tooth width $[\mathrm{mm}]$ & Number of teeth \\
\hline 100 & 2.5 & 5.96 & 19 \\
90 & 2.5 & 5.00 & 22 \\
80 & 2.5 & 4.20 & 27 \\
\hline
\end{tabular}

When the angle is $90^{\circ}$ and $100^{\circ}$, the number of teeth that can be machined on the slip anchor is too small. Combining the single tooth shear strength with the number of teeth that can be machined, the optimum thread angle of the tooth is determined to be $80^{\circ}$. After determining the thread angle, the tooth width was determined to be $4.2 \mathrm{~mm}$, and the lengths of slip anchors were corrected to be $113.4 \mathrm{~mm}$.

\section{Determinations of the parameters to be optimized.}

The parameters to be optimized are the wedge angle of the inner and outer slip anchors as well as the front and rear angles combination of the teeth. Three wedge angles of $13^{\circ}, 14^{\circ}$ and $15^{\circ}$ are chosen to optimize, and three front and rear angles combinations of $70^{\circ}$ and $10^{\circ}, 65^{\circ}$ and $15^{\circ}, 60^{\circ}$ and $20^{\circ}$ are chosen to optimize.

Determination of the jacking allowance. The setting of the jacking allowance is to ensure a sufficient jacking distance between the outer slip anchor and the baffle after completion of clamp centering so as to ensure the biting process. The jacking allowances required for all parameters are calculated in Table 2, and the maximum value is selected to design the jacking allowance. 
Table 2 The required jacking allowance for a single slip anchor

\begin{tabular}{cccc}
\hline Front angle $\left[{ }^{\circ}\right]$ & Rear angle $\left[{ }^{\circ}\right]$ & Wedge angle $\left[{ }^{\circ}\right]$ & Jacking allowance $[\mathrm{mm}]$ \\
\hline 70 & 10 & 13 & 1.45 \\
70 & 10 & 14 & 1.34 \\
70 & 10 & 15 & 1.25 \\
65 & 15 & 13 & 1.76 \\
65 & 15 & 14 & 1.63 \\
65 & 15 & 15 & 1.51 \\
60 & 20 & 13 & 2.02 \\
60 & 20 & 14 & 1.87 \\
60 & 20 & 15 & 1.74 \\
\hline
\end{tabular}

In Table 2, the maximum jacking allowance for a single slip anchor is $2.02 \mathrm{~mm}$. Both the inner and outer slip anchors need jacking allowance, so the total required jacking allowance is $4.04 \mathrm{~mm}$. At last the jacking allowance of the locking mechanism is determined to be $7 \mathrm{~mm}$, which is bigger than $4.04 \mathrm{~mm}$.

\section{Finite element analysis settings}

The Workbench of ANSYS was used to build the finite element model, and the locking mechanism was simplified as a plane strain problem.

Constraint and load settings. As shown in Figure 5, the upper part of the pipe wall and the lower part of the clamp housing wall are applied to full restraint. The left side of the inner slip anchor is subjected to compression only support to simulate the radial guide action of the baffle, and the right side of the outer slip anchor is applied with a uniform pressure of $15 \mathrm{MPa}$.

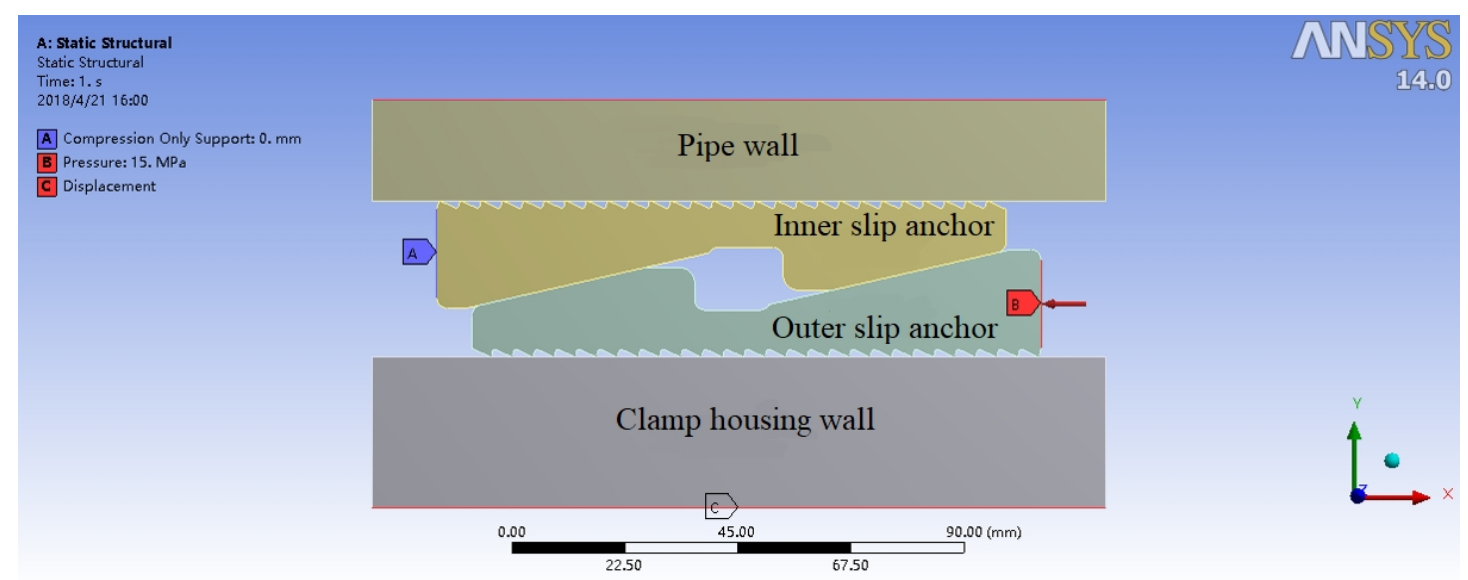

Figure 5 Constraint and load settings of the FE model

Contact settings. The contact setting of the model is shown in Fig. 6. The friction coefficients between the inner slip anchor tooth and the pipe wall, between the outer slip anchor tooth and the clamp housing wall are set to be 0.2 . The tooth is the contact surface and the wall is the target surface. The friction coefficient of the inner and outer slip anchors is set to be 0.1 . 


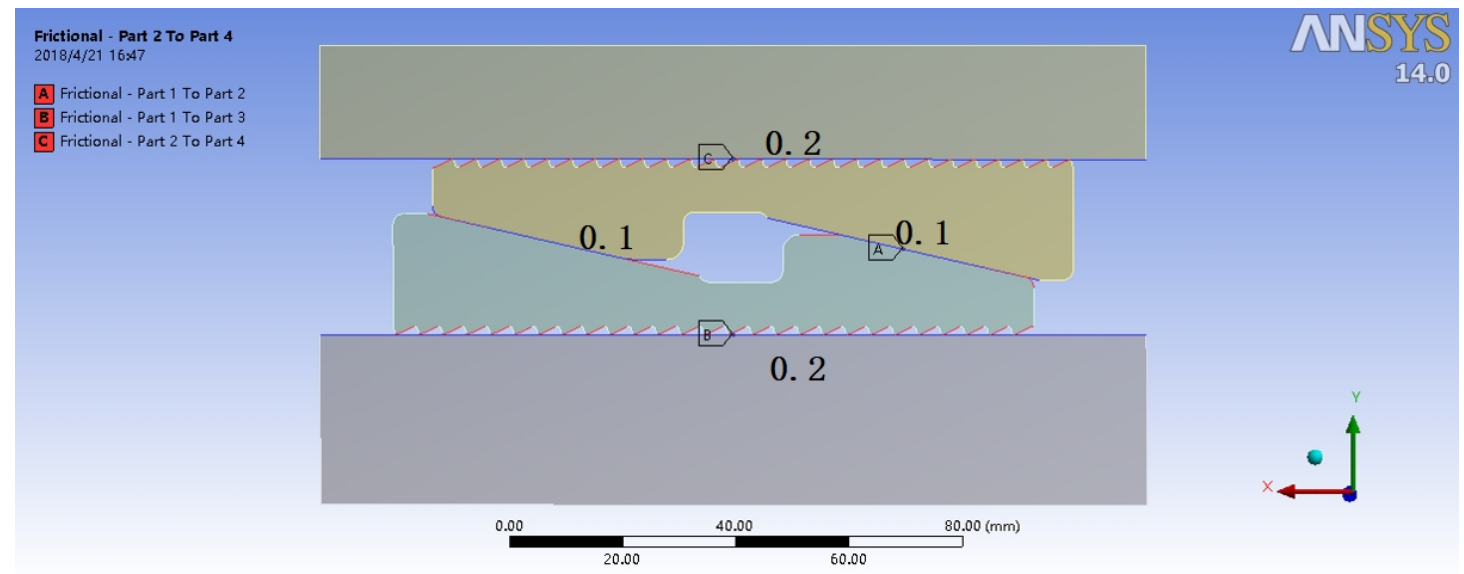

Figure 6 Contact settings of the FE model

Mesh settings. The mesh is divided according to the geometry and contracts of the model. The overall mesh size is set to be $0.4 \mathrm{~mm}$, and edge size of the pipe wall side and the clamp housing wall side in contact with the teeth is $0.2 \mathrm{~mm}$. Level 3 mesh refinement is applied at the edge of the teeth, also applied at the pipe wall side and the clamp housing wall side in contact with the teeth. The local meshing of a tooth is shown in Fig. 7.

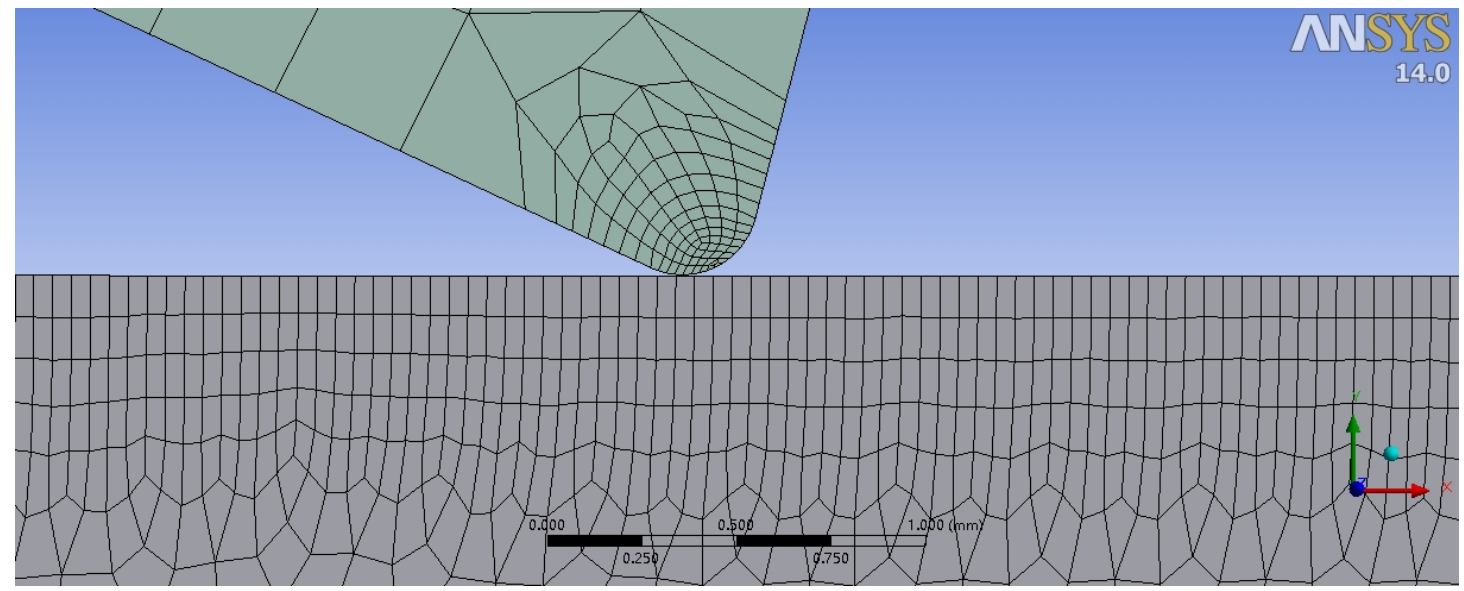

Figure 7 The enlarged view of the local mesh of a tooth

\section{Optimization of parameters}

Static analysis on the locking mechanism was performed for each design scheme. The equivalent stress of each tooth extracted by the probe was used as the contact stress of each tooth.

Wedge angle. Based on the principle of the contact stress uniformity of the teeth, an optimal wedge angle is chosen from $13^{\circ}, 14^{\circ}$ and $15^{\circ}$ respectively. The comparison of the contact stress of each tooth under each wedge angle is shown in Figure 8 and Figure 9, and the standard deviation of the contact stress of each tooth is shown in Table 3. 


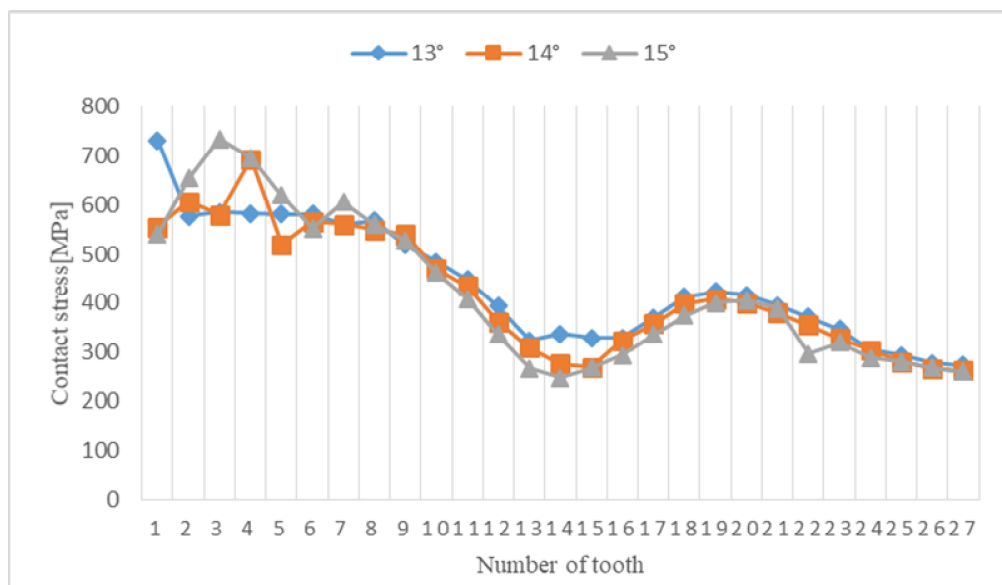

Figure 6 The contact stress of each tooth of inner slip anchor under each wedge angle

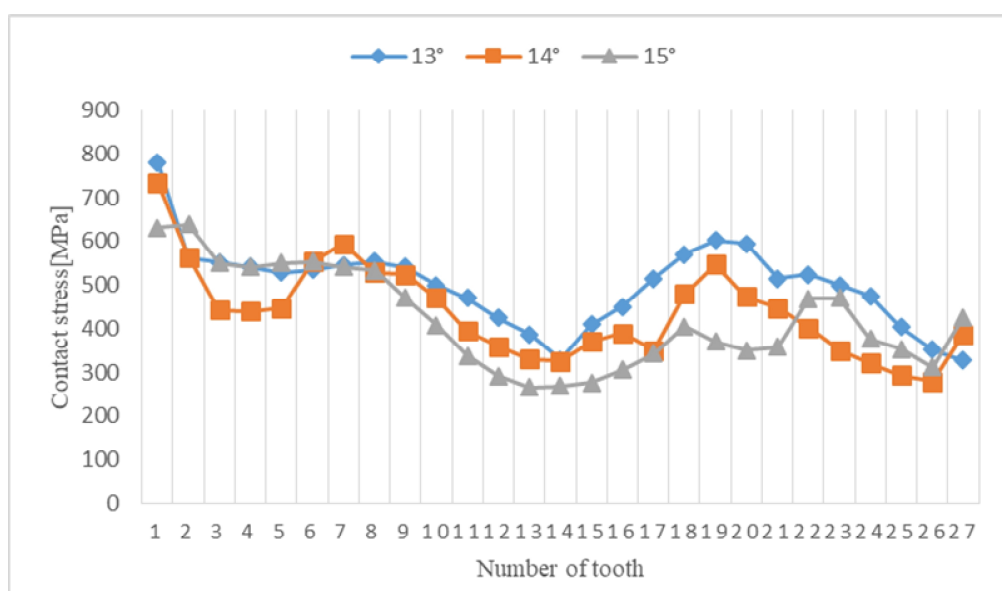

Figure 7 The contact stress of each tooth of outer slip anchor under each wedge angle

Table 3 The standard deviation of the contact stress of each tooth

\begin{tabular}{ccc}
\hline Wedge angle $\left.{ }^{\circ}\right]$ & Component & Standard deviation [MPa] \\
\hline 13 & Inner slip anchor & 119.9 \\
13 & Outer slip anchor & 94.6 \\
14 & Inner slip anchor & 122.6 \\
14 & Outer slip anchor & 103.9 \\
15 & Inner slip anchor & 147.5 \\
15 & Outer slip anchor & 111.0 \\
\hline
\end{tabular}

According to the data in the figures and Table 3, under the $13^{\circ}$ wedge angle, the uniformity of the contact stress of each tooth is better than $14^{\circ}$ and $15^{\circ}$. So the optimal wedge angle is determined to be $13^{\circ}$.

Front and rear angles combination. Based on the principle of the contact stress uniformity of the teeth, an optimal front and rear angles combination is chosen from $70^{\circ}$ and $10^{\circ}, 65^{\circ}$ and $15^{\circ}, 60^{\circ}$ and $20^{\circ}$ respectively. The comparison of the contact stress of each tooth under each combination is shown in Figure 10 and Figure 11. The standard deviation of the contact stress of each tooth is shown in Table 4. 


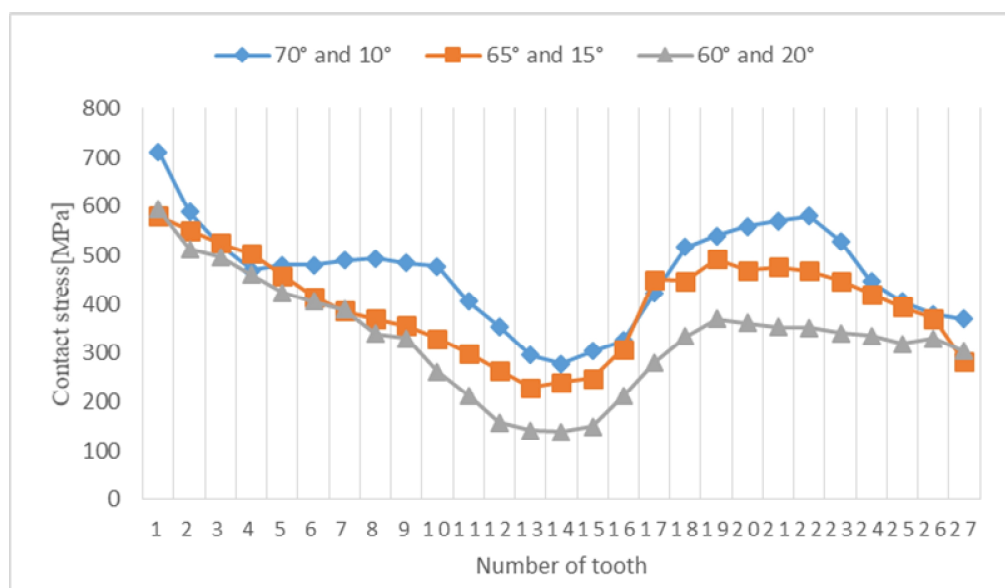

Figure 8 The contact stress of each tooth of inner slip anchor under each combination

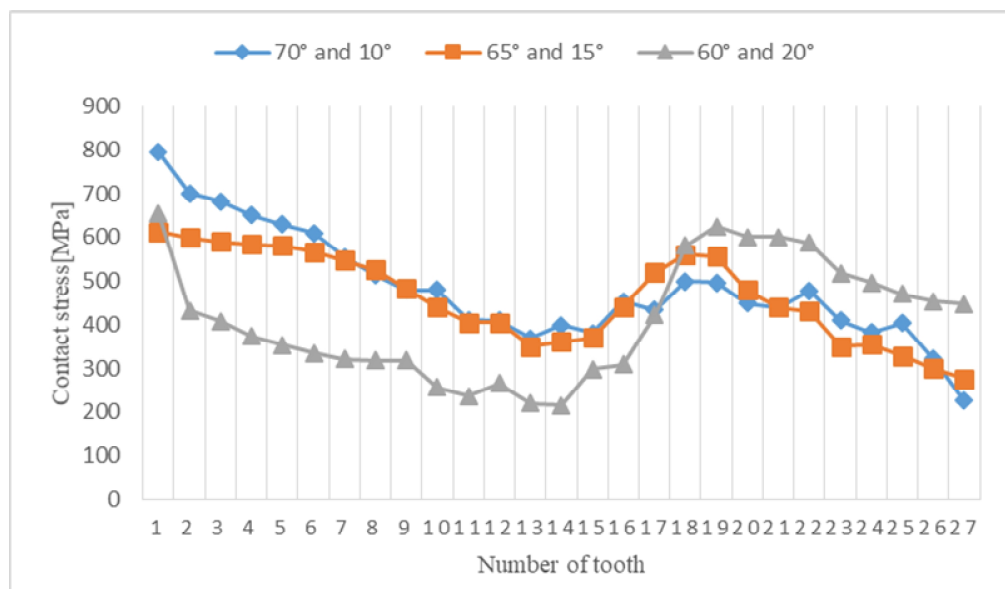

Figure 9 The contact stress of each tooth of outer slip anchor under each combination

Table 4 The standard deviation of the contact stress of each tooth

\begin{tabular}{cccc}
\hline Front angle $\left[^{\circ}\right]$ & Rear angle $\left[^{\circ}\right]$ & Component & Standard deviation $[\mathrm{MPa}]$ \\
\hline 70 & 10 & Inner slip anchor & 100.6 \\
70 & 10 & Outer slip anchor & 124.5 \\
65 & 15 & Inner slip anchor & 96.9 \\
65 & 15 & Outer slip anchor & 100.7 \\
60 & 20 & Inner slip anchor & 112.0 \\
60 & 20 & Outer slip anchor & 132.1 \\
\hline
\end{tabular}

According to the data in the figures and Table 4, under the front and rear angles combination of $65^{\circ}$ and $15^{\circ}$, the uniformity of the contact stress of each tooth is better than $70^{\circ}$ and $10^{\circ}, 60^{\circ}$ and $20^{\circ}$. So the optimal front and rear angles combination is determined to be $65^{\circ}$ and $15^{\circ}$.

\section{Conclusions}

In this paper, through the analysis on the bite-in mechanics and the failure mode of the locking mechanism of clamp, three principles for design of the locking mechanism are proposed. Then using related principles and finite element analysis, the structural parameters of the locking mechanism are optimized.

1. The analysis on the biting mechanics and the failure mode of the locking mechanism are completed, and three principles for design of the locking mechanism, which are the principle of the contact stress uniformity of the teeth, the principle of axial bearing capacity and the principle of sufficient jacking allowance, are proposed;

2. Combined with the single tooth shear strength and the number of teeth that can be machined, the optimal thread angle of the tooth is determined to be $80^{\circ}$; 
3. Based on the finite element analysis, with the principle of the contact stress uniformity of the teeth, the optimal wedge angle of the locking mechanism is determined to be $13^{\circ}$; and the optimal front and rear angles combination is determined to be $65^{\circ}$ and $15^{\circ}$.

\section{References}

[1] Zhao Fuchen, Zhang Jiahui, Ma Jinlin, Liu Kai, Fang Liang. Submarine Pipeline Maintenance Replacement Technology and Engineering Application. Shipbuilding of China, 2015, 56(Supplement 2): 390-393. (In Chinese)

[2] Du Shaoze, Zhang Zheng, Liang Jianxing. Exploring the methods of repairing submarine pipelines. Research on Urban Construction Theory, 2013, 29(1): 142-144. (In Chinese)

[3] Meng Junzheng, Rapid blockage of oil and gas pipeline leakage[J]. Oil and Gas Field Engineering, 2009, 28(7):83-84. (In Chinese)

[4] Keijser E, Bo P, Robles M, Mota H. Innovative Method for Creating New Deepwater Tie-in Points on Existing Pipeline System, Using Proven Pipeline Repair Equipment. Offshore Technology Conference, OTC 22181-MS, 2011.

[5] Ma Hongxin, Example of Leakage Repair of Ultra-Hundred Meters of Deep Seabed Oil Pipeline in South China Sea. Offshore Oil, 2010, 30(3):93-96. (In Chinese)

[6] Peng Fei, Duan Menglan, Fan Jiayu, et al. Design and Simulation of Deepwater Connector Locking Mechanism[J]. Mechanical Design \& Manufacture, 2014(1):37-39. (In Chinese)

[7] Vagata A, Reid BJ, Galletti R, Scarpa M. Diverless Special Operations. Offshore Technology Conference, OTC 21616-MS, 2011.

[8] Han Guangyao, Zhang Xiaocai, Chen Xian, Tian Zhihua. Studies on Biting Characteristic of Down-hole Tools with Slips[J]. Oil Field Equipment, 2014,43(05):49-52. (In Chinese) 